\title{
Oscilações climáticas e suas tendências para Lagoa Seca - Paraíba, Brasil
}

\author{
Climatic fluctuations and trends for Lagoa Seca - Paraíba, Brazil \\ Fluctuaciones y tendencias climáticas para Lagoa Seca - Paraíba, Brasil
}

Raimundo Mainar de Medeiros ORCID: https://orcid.org/0000-0001-7361-1281 Universidade Federal Rural de Pernambuco, Brasil E-mail: mainarmedeiros@gmail.com Wagner Rodolfo de Araújo ORCID: https://orcid.org/0000-0001-7203-0338 Universidade Estácio de Sá, Brasil E-mail: wagneraraujops@gmail.com

Romildo Morant de Holanda

ORCID: https://orcid.org/0000-0001-7945-3616 Universidade Federal Rural de Pernambuco, Brasil E-mail: romildomorant@gmail.com

Luciano Marcelo Fallé Saboya

ORCID: https://orcid.org/0000-0002-7586-6867 Universidade Federal de Campina Grande, Brasil E-mail: 1saboya@hotmail.com

Manoel Viera de França

ORCID: https://orcid.org/0000-0003-4973-9327 Universidade Federal Rural de Pernambuco, Brasil E-mail: manoelvieira.ufrpe@gmail.com

Fernando Cartaxo Rolim Neto

ORCID: https://orcid.org/0000-0002-6411-2058 Universidade Federal Rural de Pernambuco, Brasil E-mail: fernandocartaxo@yahoo.com.br

\begin{abstract}
Resumo
Objetiva-se analisar as oscilações das tendências climáticas temporais de precipitação e temperatura entre 1981-2019 para a cidade de Lagoa Seca buscando possíveis avarias destas oscilações nas atividades socioeconômicas, auxiliando os tomadores de decisão a respeito dos riscos possíveis. Os dados climáticos de precipitação foram adquiridos da Agencia executiva d'água e clima do Estado da Paraíba referentes a 1981 a 2019 e os dados térmicos foi estimado pelo software Estima_T referente ao mesmo período de estudo. As irregularidades pluviais entre 2000-2019 registraram dez anos seco; seis anos muito seco; três anos muito chuvoso e um ano chuvoso, estas irregularidades pluviais nos leva a concluir que eventos climáticos e as ações antrópicas podem estar contribuindo para os registros destes cenários em escala local, a predominância de anos seco e muito seco vem ocasionando impactos aos produtores do hortifrutigranjeiro que vem utilizando-se de maiores tempos em irrigações. Estudos com tendências climáticas tornam-se mais compreensível quando se analisar séries temporais longas as quais possam ser divididas em subperíodos de 30 anos ao menos. As anomalias pluviais entre 2000-2019 registraram dez anos seco; seis anos muito seco; três anos muito chuvoso e um ano chuvoso, estas irregularidades pluviais nos leva a concluir que eventos climáticos e as ações antrópicas podem estar contribuindo para os registros destes cenários em escala local, a predominância de anos seco e muito seco vem ocasionando impactos aos produtores do hortifrutigranjeiro que vem utilizando-se de maiores tempos em irrigações.
\end{abstract}

Palavras-chave: Índices pluviais e térmicos; Variabilidades e mudanças climáticas; Irregularidades pluviais.

\begin{abstract}
The objetive is to analyze the fluctuations of temporal climate trends of precipitation and temperature between 19812019 for the city of Lagoa Seca, looking for possible damages of these fluctuations in socioeconomic activities, helping decision makers regarding possible risks. The climatic precipitation data were acquired from the Executive Agency of Water and Climate of the State of Paraíba for 1981 to 2019 and the thermal data was estimated by the Estima_T software for the same period of study. Rainfall irregularities between 2000-2019 registered ten dry years; six years too dry; three very rainy years and one rainy year, these rainfall irregularities lead us to conclude that climatic events and anthropogenic actions may be contributing to the records of these scenarios at a local scale, the predominance of dry and very dry years has impacted produce and produce which has been using longer times in irrigation. Studies with climate trends become more understandable when analyzing long time series which can be
\end{abstract}


divided into sub-periods of at least 30 years. Rainfall anomalies between 2000-2019 recorded ten dry years; six years too dry; three very rainy years and one rainy year, these rainfall irregularities lead us to conclude that climatic events and anthropogenic actions may be contributing to the records of these scenarios at a local scale, the predominance of dry and very dry years has impacted produce and produce which has been using longer times in irrigation.

Keywords: Rain and thermal indices; Variability and climate change; Rain irregularities.

\section{Resumen}

El objetivo es analizar las fluctuaciones de las tendencias climáticas temporales de precipitación y temperatura entre 1981-2019 para la ciudad de Lagoa Seca, buscando posibles daños de estas fluctuaciones en las actividades socioeconómicas, ayudando a los tomadores de decisiones sobre posibles riesgos. Los datos de precipitación climática fueron adquiridos de la Agencia Ejecutiva de Agua y Clima del Estado de Paraíba de 1981 a 2019 y los datos térmicos fueron estimados por el software Estima_T para el mismo período de estudio. Las irregularidades de las precipitaciones entre 2000-2019 registraron diez años secos; seis años demasiado secos; tres años muy lluviosos y un año lluvioso, estas irregularidades pluviales nos llevan a concluir que eventos climáticos y acciones antropogénicas pueden estar contribuyendo al registro de estos escenarios a escala local, el predominio de años secos y muy secos ha impactado productos y productos que ha estado utilizando tiempos más largos en riego. Los estudios con tendencias climáticas se vuelven más comprensibles cuando se analizan series de tiempo largas que se pueden dividir en subperíodos de al menos 30 años. Las anomalías de precipitación entre 2000-2019 registraron diez años secos; seis años demasiado secos; tres años muy lluviosos y un año lluvioso, estas irregularidades pluviales nos llevan a concluir que eventos climáticos y acciones antropogénicas pueden estar contribuyendo al registro de estos escenarios a escala local, el predominio de años secos y muy secos ha impactado productos y productos que ha estado utilizando tiempos más largos en riego.

Palabras clave: Precipitaciones e índices térmicos; Variabilidad y cambio climático; Irregularidades de lluvia.

\section{Introdução}

Nas últimas duas décadas as comunidades cientificas e acadêmicas vêm debatendo sobre as mudanças climáticas (IPCC, 2014; Andrade et al., 2014; Nunes, 2016; Wanderley et al., 2018). Isso vem ocorrendo devido às alterações dos cenários nas mudanças climáticas acontecidas, a qual registra acréscimo de $2{ }^{\circ} \mathrm{C}$ na temperatura global do planeta, abrangendo grandes desequilíbrios em ecossistemas, como movimentos expressivas na Floresta Amazônica afetando toda sua biodiversidade, acarretado perdas expressivas de geleiras nas cordilheiras dos Andes e Himalaia, em conformidade ao estudo do Primeiro Relatório de Avaliação Nacional do Painel Brasileiro de Mudanças Climáticas (PBMC, 2014).

Penereiro et al. (2013) revelara que os diagnósticos espaciais de tendências climáticas consentem a observação das oscilações no comportamento das suas alterações, fornecendo esclarecimento de subsídios de uma variável em uma região que vem permitindo mudanças significativas ao longo do tempo. Estudar as alterações climáticas ocorridas no passado permite uma melhor compreensão das variabilidades induzidas no presente, além de subsidiar elementos para investigação do comportamento do clima futuro.

Medeiros et al. (2018) estudaram as variabilidades pluviais para São Bento do Una (PE), forte centro avícola, em razão ao aumento da demanda hídrica necessária ao pleno desenvolvimento de suas atividades. O estudo pode ser utilizado como ferramenta para planejamentos e ações que visem gerenciar os recursos hídricos utilizando sistemas de captação d'água, armazenamento e represamento, evitando a problemática da escassez de água. Ressaltaram ainda, que existe a necessidade de políticas e planos de captação e aproveitamento das águas das chuvas, além do uso eficiente dos demais recursos naturais da região, para que o desenvolvimento socioeconômico não seja limitado pela baixa disponibilidade hídrica.

Medeiros et al, (2017) utilizaram-se das alterações pluviais mensais e verificaram sua possível tendência linear, identificando-se as maiores variabilidades na precipitação de Serra Talhada (PE) registra-se entre janeiro a abril, registrando altos índices pluviais, seus índices com menores valores achar entre julho a outubro. Contudo, os autores afirmaram que a pluviosidade local é bastante irregular no espaço, tempo e quantidade, sua distribuição e duração acontecem de forma diferenciada. 
Priori (2014) mostrou que em áreas de ecossistemas frágeis e vulneráveis, como a região semiárida brasileira, onde as mudanças climáticas podem ocasionar marés altas ou tempestades severas em curto intervalo de tempo irá dificultar a drenagem das águas para o mar e causar o prolongamento de cheias, enchentes e/ou inundações causando problemas de saúde e socioeconômica à população local. Silva et al. (2008) advertiram que danos econômicos a agropecuária com perdas parciais ou totais poderá acontecer, afetando o comprometendo e os abastecimentos d'água para a população ocasionado pelas altas irregularidades pluviais, com predominância de chuvas intensas e de curta duração, causando forte impacto social aos habitantes da região.

OPAS (2014) esclareceu que a velocidade e a magnitude nas mudanças poderão causar à extinção de muitas espécies (fauna e flora), acarretando em alterações ambientais que levara ao agravamento dos desastres como aumento do impacto socioeconômico e cultural, o crescimento populacional, urbanização, industrialização, o consumo de recursos naturais e da demanda sobre os ciclos biogeoquímicos. O acréscimo das concentrações de gases do efeito estufa na atmosfera, que são intensificados pelas atividades humanas (Ghini et al., 2008), causado pela soma das ações produzidas pelas concentrações de gases de efeito estufa (Santos, 2006).

Santos Neto et al. (2014) asseguraram que entender o comportamento da tendência sazonal dos índices pluviométricos servem como opção para resolver múltiplos problemas de planejamentos urbano e rural, recursos hídricos, agropecuários, industriais e hortifrutigranjeiros, serviços de alertas a catástrofes e condicionar o cotidiano das atividades humanas locais.

Medeiros et al. (2016) analisando o comportamento das tendências térmicas e pluviais nas condições climatológicas em Bom Jesus - PI, salientaram que o cenário futuro é pessimista, sugerindo uma redução de até $20 \%$ na precipitação e $4{ }^{\circ} \mathrm{C}$ de aumento na temperatura.

Estudos que mostraram possíveis tendências temporais de temperatura (Medeiros et al., 2018), precipitação (Groppo et al., 2008; Amazon et al., 2012; Karmeshu, 2012; Wanderley et al., 2018; Yang et al. 2010; Chierice et al. (2014); Ishihara et al. 2014; Nóbrega et al. 2015; Lai et al. 2016 \& Zhijia et al. 2016). (Farias et al., 2012; Duarte et al., 2015) trabalharam com eventos extremos de precipitação a partir da técnica dos Quantis para o estado Pernambucano visando relacioná-los aos desastres decorrentes das tendências temporais

Lagoa Seca - PB tem como uma da fonte de sobrevivência humana o desenvolvimento da produtividade da hortifrutigranjeiro, este artigo tem a intensão de chamar a atenção aos poderes governamentais municipal e estadual para a realização de melhores projetos visando às ocorrências dos efeitos ou das ocorrências dos eventos extremos que poderá vim acontecer causando prejuízos aos hortifrutigranjeiros e a socioeconômica.

Objetiva-se analisar as oscilações das tendências climáticas temporais de precipitação e temperatura entre 1981-2019 para a cidade de Lagoa Seca (PB) buscando possíveis avarias destas oscilações nas atividades socioeconômicas e fornecendo subsídios aos tomadores de decisão a respeito dos riscos possíveis.

\section{Material e Métodos}

Lagoa seca localiza-se na latitude de $07^{\circ} 10^{\prime} 15^{\prime \prime} \mathrm{S}$; longitude de 35\%51'13', W e 634 metros de altitude, posicionam-se na Microrregião de Lagoa seca e Mesorregião do Agreste Paraibano. Limita-se com: Campina Grande, Massaranduba, Matinhas, São Sebastião de Lagoa de Roça, Montadas, Puxinanã e Esperança. (Figura 1). 
Figura 1. Posicionamento da cidade de Lagoa Seca dentro do estado da Paraíba.

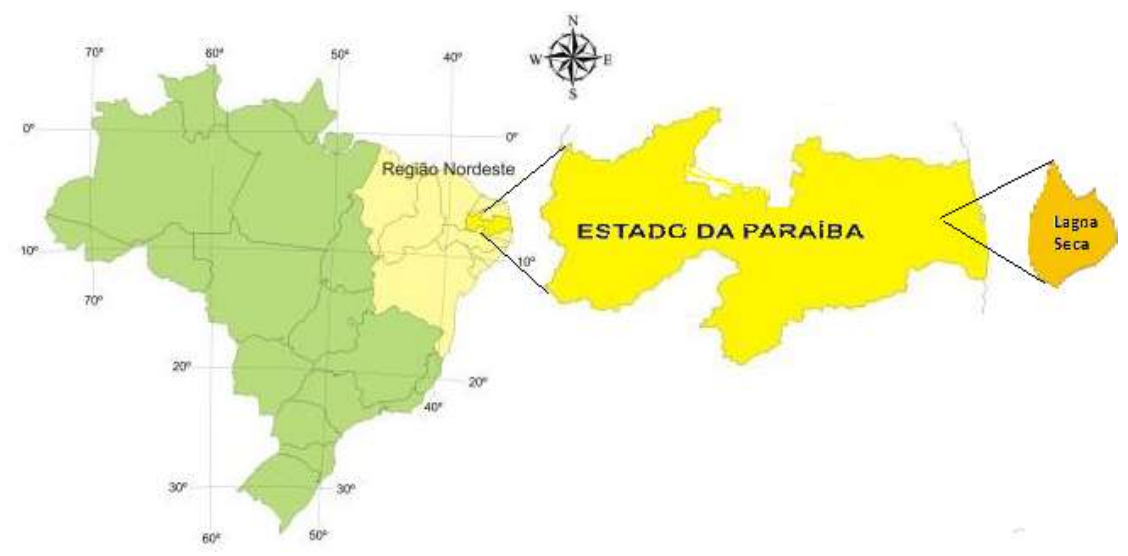

Fonte: Medeiros (2021).

Segundo (Köppen 1928; Köppen et al., 1931) o clima é do tipo “As” (quente e úmido tropical chuvoso). Estudos como o de Alvares et al. (2014) corroborar com o tipo de clima estudado. A classificação climática de (Thornthwaite 1948; Thornthwaite et al., 1955) é do tipo ( $\mathrm{C}_{1} \mathrm{ADa}$ ') seco subúmido, megatérmico, com pequena ou nenhum excesso d'água e evapotranspiração com 29,66\% da evapotranspiração potencial anual concentrada no trimestre mais quente do ano (novembro, dezembro e janeiro). As amplitudes térmicas têm as suas variações com a latitude, altitude e com o grau de continentalidade (efeitos de montanhas, orografia; vales, morros, etc.).

O regime pluvial insere-se na faixa das isoietas (linha que une o mesmo valor de precipitação) de $1.100 \mathrm{~mm}^{\mathrm{ano}}{ }^{1} \mathrm{a}$ 1.200 mm.ano ${ }^{1}$. (Medeiros, 2016). As chuvas têm início por volta da segunda quinzena de março, aumentando de volume nos primeiros dias de abril e se prolonga até agosto, sendo o trimestre mais chuvoso os meses de maio a julho. No município estudado especificamente, as chuvas são fundamentais para o bom desenvolvimento do regime dos rios perenes, córregos, riachos, níveis dos lagos e lagoas, bem como para a ocupação do solo, sendo imprescindível ao planejamento de qualquer atividade o conhecimento da sua dinâmica. As complementações dos fatores provocadores de chuva no município são formações de linhas de instabilidade na costa e transportada para o interior pelos ventos alísios de nordeste, desenvolvimento de aglomerados convectivos, proveniente do calor armazenado na superfície e transferido para atmosfera, orografia, contribuições das formações dos vórtices ciclônicos, e tendo como principal sistema o posicionamento da Zona de Convergência Intertropical. Normalmente as chuvas têm intensidade moderada seguidas de irregularidade devidas ás falhas dos sistemas meteorológicos atuantes. Salienta-se que a ocorrência de períodos de veranicos (ocorrências de vários dias consecutivos sem chuva dentro do período chuvoso) no quadrimestre chuvoso (abril a julho) é possível e variante de ano para ano. Sua magnitude é variada dependendo da época e dos fatores meteorológicos. Tem-se registrado ocorrências com períodos de veranicos superiores há 17 dias mensais no intervalo de tempo decorrido dentro do quadrimestre. (Medeiros, 2016).

Os dados pluviais foram fornecidos pela Agência executiva d'água e clima do Estado da Paraíba (AESA, 2020), entre 1981 a 2019. Os dados da temperatura são referentes ao mesmo período dos dados pluviais.

Utilizou-se do Software estima t_para o cálculo da temperatura levando em consideração as coordenadas geográficas do local e utilizando-se da equação seguinte.

$$
\mathrm{T}=\mathrm{C}_{0}+\mathrm{C}_{1} \lambda+\mathrm{C}_{2} \varnothing+\mathrm{C}_{3} \mathrm{~h}+\mathrm{C}_{4} \lambda^{2}+\mathrm{C}_{5} \emptyset^{2}+\mathrm{C}_{6} \mathrm{~h}^{2}+\mathrm{C}_{7} \lambda \varnothing+\mathrm{C}_{8} \lambda \mathrm{h}+\mathrm{C}_{9} \varnothing \mathrm{h}
$$

Em que:

$\mathrm{C}_{0}, \mathrm{C}_{1}, \ldots ., \mathrm{C}_{9}$ constantes;

$\lambda, \lambda^{2}, \lambda \varnothing, \lambda \mathrm{h}$ longitude;

$\varnothing, \varnothing^{2}, \lambda \varnothing$ latitude; $h, h^{2}, \lambda \mathrm{h}$

$\varnothing \mathrm{h}$ altura. 
Os dados trabalhados têm 33 anos de observações continuas e segundo o World Metereological Organization (WMO, 2019) o clima de dada área ou região é caracterizado por um período mínimo de 30 anos com observações continuas, pois quanto maior o período dos dados, maior será a credibilidade da distinção climática da área estudada. Estas apresentam séries históricas longas suficientes para serem classificados quanto à variabilidade climática, e são distribuídas uniformemente.

Os dados foram preparados em planilhas eletrônicas pelo Microsoft Office Excel 2016, gerando-se gráficos e tabelas, realizaram suas análises para a precipitação, temperatura média e ocorrência de tendência por utilização dos parâmetros citados.

Os dados de precipitação foram totalizados anualmente e, em seguida, foram calculados os quantis similarmente aos utilizados por (Pinkayan, 1966; Xavier e Xavier, 1987; Xavier, 2002), referentes às probabilidades $\left(\mathrm{Q}_{0,15}\right),\left(\mathrm{Q}_{0,35}\right)$, $\left(\mathrm{Q}_{0,65}\right)$ e $\left(\mathrm{Q}_{0,85}\right)$, de forma que, para cada ano, considerou-se os dados pluviais, (Tabela 1). E na (Tabela 2) tem-se os cálculos dos quantis mensais e suas referentes probabilidades.

Tabela 1. Classificação, probabilidade e intervalo médio de precipitação anual $(\mathrm{mm})$ dos regimes pluviométricos de 1981 a 2019 para Lagoa Seca - Paraíba.

\begin{tabular}{cccc}
\hline Classificação & Probabilidade & Intervalo médio de precipitação $(\mathrm{mm})$ \\
& & Mínimo & máximo \\
\hline Muito seco & $\mathrm{P}<\mathrm{Q}_{(0,15)}$ & - & 922,90 \\
Seco & $\mathrm{Q}_{(0,15) \leq} \mathrm{P}_{<} \mathrm{Q}_{(0,35)}$ & 922,90 & 1083,25 \\
Normal & $\mathrm{Q}_{(0,35) \leq} \mathrm{P}_{<} \mathrm{Q}_{(0,65)}$ & 1083,25 & 1210,79 \\
Chuvoso & $\mathrm{Q}_{(0,65) \leq} \mathrm{P}_{<} \mathrm{Q}_{(0,85)}$ & 1210,79 & 1294,79 \\
Muito chuvoso & $\mathrm{P}>\mathrm{Q}_{(0,85)}$ & 1294,79 & - \\
\hline
\end{tabular}

Fonte: Xavier e Xavier (1987).

Tabela 2. Classificação, probabilidade e intervalo médio de precipitação mensal (mm) dos regimes pluviais de 1981 a 2019 para de Lagoa Seca - Paraíba.

\begin{tabular}{cccc}
\hline Classificação & Probabilidade & $\begin{array}{c}\text { Intervalo médio de precipitação }(\mathrm{mm}) \\
\text { Mínimo }\end{array}$ & máximo \\
\hline Muito seco & $\mathrm{P}<\mathrm{Q}(0,15)$ & $<306,9$ & 307,0 \\
Seco & $\mathrm{Q}(0,15) \leq \mathrm{P}<\mathrm{Q}(0,35)$ & 307,1 & 361,1 \\
Normal & $\mathrm{Q}(0,35) \leq \mathrm{P}<\mathrm{Q}(0,65)$ & 361,2 & 403,6 \\
Chuvoso & $\mathrm{Q}(0,65) \leq \mathrm{P}<\mathrm{Q}(0,85)$ & 403,7 & 431,6 \\
Muito chuvoso & $\mathrm{P}>\mathrm{Q}(0,85)$ & 431,7 & $<431,8$ \\
\hline
\end{tabular}

Fonte: Adaptada por Medeiros (2021).

\section{Resultados e Discussão}

Na Tabela 3 têm-se as flutuações pluviais anuais, suas classificações conforme os intervalos dos quantis para cada ano, os anos classificados como "seco" foram: 1980; 1991; 1995; 1997 a 1999; 2001-2003; 2005; 2008; 2010; 2013; 2014; 2018 e 2019.

Os anos classificados como "muito seco" foram 1981; 1982; 1993; 2006; 2007; 2012; 2015 e 2017. Os anos classificados como "normal pluviométrico" foram: 1983; 1984; 1990 e 1996. Os anos de 1987; 1988 e 2009 comportaram-se como ano "chuvoso". Registrou-se categoria "muito chuvosa” para os anos: 1985; 1986; 1992; 1994; $2000 ; 2004$ e 2011. 
Tabela 3. Anos, total anual pluvial, classificação conforme os intervalos dos quantis para Lagoa Seca - PB entre 1981-2019.

\begin{tabular}{|c|c|c|c|c|c|}
\hline Ano & Total Anual & Classificação & Ano & Total Anual & Classificação \\
\hline 1981 & 856,0 & Muito seco & 2001 & 1042,5 & Seco \\
\hline 1982 & 867,0 & Muito seco & 2002 & 1010,8 & Seco \\
\hline 1983 & 1091,1 & Normal & 2003 & 998,7 & Seco \\
\hline 1984 & 1182,2 & Normal & 2004 & 1697,7 & Muito chuvoso \\
\hline 1985 & 1657,0 & Muito chuvoso & 2005 & 971,5 & Seco \\
\hline 1986 & 1522,4 & Muito chuvoso & 2006 & 783,1 & Muito seco \\
\hline 1987 & 1236,7 & Chuvoso & 2007 & 844,2 & Muito seco \\
\hline 1988 & 1251,5 & Chuvoso & 2008 & 1078,7 & Seco \\
\hline 1989 & 928,5 & Seco & 2009 & 1256,4 & Chuvoso \\
\hline 1990 & 1088,8 & Normal & 2010 & 971,4 & Seco \\
\hline 1991 & 1078,0 & Seco & 2011 & 1797,1 & Muito chuvoso \\
\hline 1992 & 1433,4 & Muito chuvoso & 2012 & 748,4 & Muito seco \\
\hline 1993 & 607,5 & Muito seco & 2013 & 994,5 & Seco \\
\hline 1994 & 1667,5 & Muito chuvoso & 2014 & 1010,6 & Seco \\
\hline 1995 & 955,4 & Seco & 2015 & 807,1 & Muito seco \\
\hline 1996 & 1202,0 & Normal & 2016 & 809,3 & Muito seco \\
\hline 1997 & 961,1 & Seco & 2017 & 856,4 & Muito seco \\
\hline 1998 & 586,0 & Seco & 2018 & 938,7 & Seco \\
\hline 1999 & 817,7 & Seco & 2019 & 1077,0 & Seco \\
\hline 2000 & 1584,4 & Muito chuvoso & & & \\
\hline
\end{tabular}

Fonte: Medeiros (2021).

Moura (2016) explicou que a ampliação urbana desordenada pode ser considerada como uma das causas do agravamento desta situação, como a ampliação dos desastres naturais (estiagens prolongadas, inundações, cheias, alagamento, enchentes, erosão, entre outros fenômenos). Segundo ainda o autor as ações voltadas à sustentabilidade, podem ajudar com melhorias necessárias nos âmbitos socioeconômico e ambiental e nas cidades afetadas para a estabilização do clima. Esta afirmação vem a corroborar com as discussões aqui apresentadas.

$\mathrm{Na}$ Tabela 4 têm-se os anos, suas classificações mensais de acordo com os intervalos dos quantis (Tabela 2) para o município de Lagoa Seca - PB no período de 1981-2019. Levando em considerações os intervalos de classes quantilicos foram determinados quatros (4) tipos de classificações. A classificação "muito seca" (MS) predominou na maioria dos meses, no mês de junho de 1994 e janeiro de 2004 registrou chuvas entre as normalidades (N), os meses de maio e julho de 2011 foram classificados como "muito chuvoso" (MC) e o mês de julho de 2015 classificou-se como "seco (S). Tais variabilidades foram decorrentes dos sistemas atmosféricos atuantes, as contribuições locais e regionais que não foram necessárias e suficientes para ocasionarem chuvas acima da média quantilicas. Estudos como o de (Marengo et al. 2008; Marengo et al. 2015) vem a contribuírem com os resultados discutidos neste artigo.

A climatologia dos eventos extremos dos valores máximos pluviais mensais mostra que as maiores frequências absolutas dos Quantis de chuva forte, concentradas entre os meses de março a setembro. Nos meses de outubro a dezembro apresentaram menor registro de chuvas mensais, muito fortes $(15<\mathrm{X}<35 \mathrm{~mm})$. 
Tabela 4. Anos, classificações mensais de acordo com os intervalos dos quantis (Tabela 2) para o município de Lagoa Seca PB no período de 1981-2019.

\begin{tabular}{|c|c|c|c|c|c|c|c|c|c|c|c|c|}
\hline $\mathrm{NO}$ & AN & EV & IAR & ABR & MAI & JUN & JUL & AGO & SET & OUT & $\mathrm{NOV}$ & DEZ \\
\hline 1981 & MS & MS & MS & MS & MS & MS & MS & MS & MS & MS & MS & MS \\
\hline 1982 & MS & MS & MS & MS & MS & MS & MS & MS & MS & MS & MS & MS \\
\hline 1983 & MS & MS & MS & MS & MS & MS & MS & MS & MS & MS & MS & MS \\
\hline 1984 & MS & MS & MS & MS & MS & MS & MS & MS & MS & MS & MS & MS \\
\hline 1985 & MS & MS & MS & MS & MS & MS & MS & MS & MS & MS & MS & MS \\
\hline 1986 & MS & MS & MS & MS & MS & MS & MS & MS & MS & MS & MS & MS \\
\hline 1987 & MS & MS & MS & MS & MS & MS & MS & MS & MS & MS & MS & MS \\
\hline 1988 & MS & MS & MS & MS & MS & MS & MS & MS & MS & MS & MS & MS \\
\hline 1989 & MS & MS & MS & MS & MS & MS & MS & MS & MS & MS & MS & MS \\
\hline 1990 & MS & MS & MS & MS & MS & MS & MS & MS & MS & MS & MS & MS \\
\hline 1991 & MS & MS & MS & MS & MS & MS & MS & MS & MS & MS & MS & MS \\
\hline 1992 & MS & MS & MS & MS & MS & MS & MS & MS & MS & MS & MS & MS \\
\hline 1993 & MS & MS & MS & MS & MS & MS & MS & MS & MS & MS & MS & MS \\
\hline 1994 & MS & MS & MS & MS & MS & $\mathrm{N}$ & MS & MS & MS & MS & MS & MS \\
\hline 1995 & MS & MS & MS & MS & MS & MS & MS & MS & MS & MS & MS & MS \\
\hline 1996 & MS & MS & MS & MS & MS & MS & MS & MS & MS & MS & MS & MS \\
\hline 1997 & MS & MS & MS & MS & MS & MS & MS & MS & MS & MS & MS & MS \\
\hline 1998 & MS & MS & MS & MS & MS & MS & MS & MS & MS & MS & MS & MS \\
\hline 1999 & MS & MS & MS & MS & MS & MS & MS & MS & $M S$ & MS & MS & MS \\
\hline 2000 & MS & MS & MS & MS & MS & MS & MS & MS & MS & MS & MS & MS \\
\hline 2001 & MS & MS & MS & MS & MS & MS & MS & MS & MS & MS & MS & MS \\
\hline 2002 & MS & MS & MS & MS & MS & MS & MS & MS & MS & MS & MS & MS \\
\hline 2003 & MS & MS & MS & MS & MS & MS & MS & MS & 10 & MS & MS & MS \\
\hline 2004 & $\mathrm{~N}$ & MS & MS & MS & MS & MS & MS & MS & MS & MS & MS & MS \\
\hline 2005 & MS & MS & MS & MS & MS & MS & MS & MS & 10 & MS & MS & MS \\
\hline 2006 & MS & MS & MS & MS & MS & MS & MS & MS & MS & MS & MS & MS \\
\hline 2007 & MS & MS & MS & MS & MS & MS & MS & MS & MS & MS & MS & MS \\
\hline 2008 & MS & MS & MS & MS & MS & MS & MS & MS & MS & MS & MS & MS \\
\hline 2009 & MS & MS & MS & MS & MS & MS & MS & MS & MS & MS & MS & MS \\
\hline 2010 & MS & MS & MS & MS & MS & MS & MS & MS & MS & MS & MS & MS \\
\hline 2011 & MS & MS & MS & MS & $\mathrm{MC}$ & MS & $\mathrm{MC}$ & MS & MS & MS & MS & MS \\
\hline 2012 & MS & MS & MS & MS & MS & MS & MS & $\mathrm{MS}$ & MS & MS & MS & MS \\
\hline 2013 & MS & MS & MS & MS & MS & MS & MS & MS & MS & MS & MS & MS \\
\hline 2014 & MS & MS & MS & MS & MS & MS & MS & MS & MS & MS & MS & MS \\
\hline
\end{tabular}




$\begin{array}{lllllllllllll}2015 & \text { MS } & \text { MS } & \text { MS } & \text { MS } & \text { MS } & \text { MS } & \text { S } & \text { MS } & \text { MS } & \text { MS } & \text { MS } & \text { MS } \\ 2016 & \text { MS } & \text { MS } & \text { MS } & \text { MS } & \text { MS } & \text { MS } & \text { MS } & \text { MS } & \text { MS } & \text { MS } & \text { MS } & \text { MS } \\ 2017 & \text { MS } & \text { MS } & \text { MS } & \text { MS } & \text { MS } & \text { MS } & \text { MS } & \text { MS } & \text { MS } & \text { MS } & \text { MS } & \text { MS } \\ 2018 & \text { MS } & \text { MS } & \text { MS } & \text { MS } & \text { MS } & \text { MS } & \text { MS } & \text { MS } & \text { MS } & \text { MS } & \text { MS } & \text { MS } \\ 2019 & \text { MS } & \text { MS } & \text { MS } & \text { MS } & \text { MS } & \text { MS } & \text { MS } & \text { MS } & \text { MS } & \text { MS } & \text { MS } & \text { MS }\end{array}$

Legenda: MS - muito seco; S - seco; N - Normal. Fonte: Medeiros (2021).

Monteiro et al. (2012) com dados correspondente à série histórica no período de 1980 a 2009 da Empresa de Pesquisa Agropecuária do Rio Grande do Norte - EMPARN, na região do baixo curso do rio Apodi-Mossoró, utilizando a técnica dos Quantis, por meio da metodologia proposta por (Pinkayan 1966; Xavier 2002; Xavier 2007), o valor aproximado mensal de chuvas foi de $642,8 \mathrm{~mm}$, sendo que os intervalos estabelecidos para cada classe extrema indicaram valores abaixo de 299,2 mm, o que corroboram com os resultados demonstrados na Tabela 4.

Wanderley et al. (2018) observaram maiores volumes diários de precipitação registrados entre 1961 e 2016 em Recife, todavia, esses dados foram diferentes ao concretizado nesse estudo, o período das chuvas para o município em estudo sofreu redução ocasionado pelo início do período seco.

Na Figura 2 observam-se a oscilação pluvial anual, média climatológica do período 1981- 2019 e seus valores quantis $\mathrm{Q}_{(0,15)}, \mathrm{Q}_{(0,35)}, \mathrm{Q}_{(0,65)}$ e $\mathrm{Q}_{(0,85)}$, para a cidade de Lagoa Seca - PB. As classificações dos anos pluviais foram realizadas de acordo com a Tabela 1, levando em considerações as classificações e as probabilidades para os intervalos médios da precipitação em relação aos quantis.

Para o $\mathrm{Q}(0,15)$ tem-se dois anos com valores anuais abaixo $(1983,1998)$; para o $\mathrm{Q}(0,35)$ apresentam os anos abaixo deste 1981, 1982, 1990, 1991, 1999, 2006, 2007, 2015 a 2017. No $Q_{(0,65)}$ registrou-se os anos de 1983, 1984, 1991, 1991, 1996, 2001 a 2003, 2005, 2008, 2010. Com índices pluviais superiores aos $Q_{(0,85)}$ foram os anos de 1985, 1986, 1994, 2000,2004 e 2011. Os anos que ficaram abaixo do $\mathrm{Q}_{(0,85)}$ foram secos conforme a distribuições quantilicas apresentadas por Xavier e Xavier (1987).

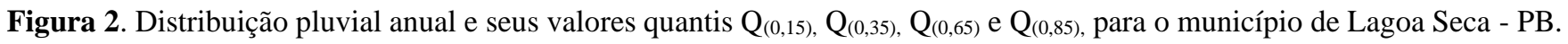

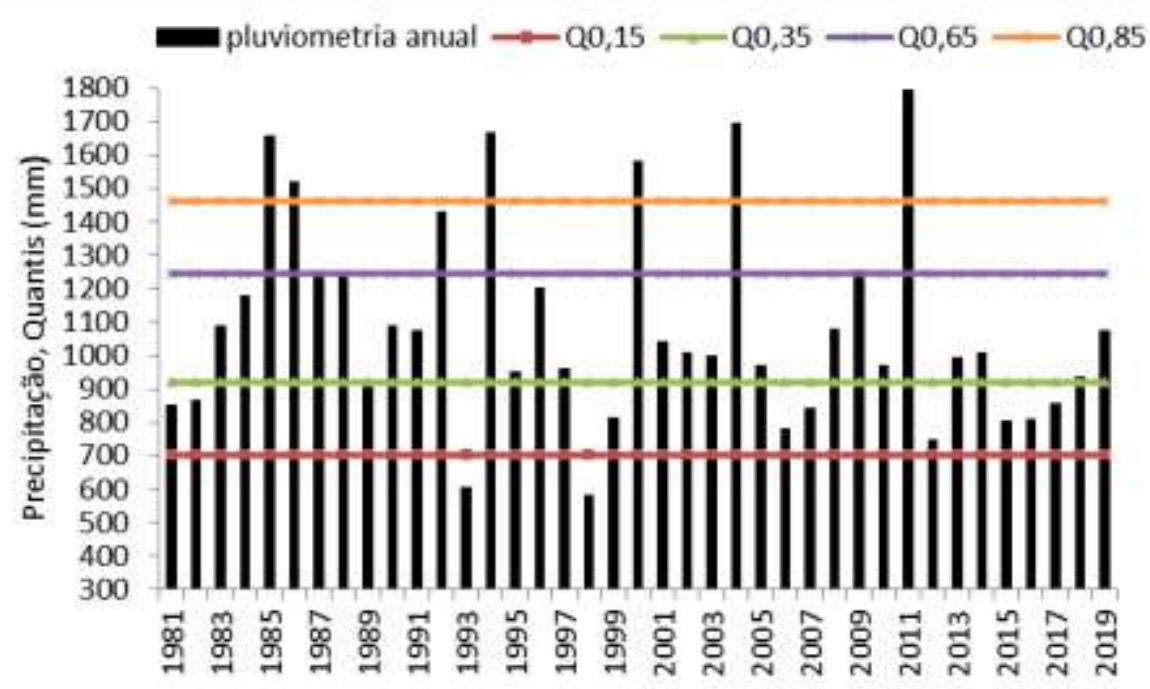

Fonte: Medeiros (2021). 
A curvatura polinomial (Figura 3) da precipitação demonstra os anos secos e chuvosos bem definidos além de mostrar que nos recentes anos compreendido entre 2010-2018 a redução dos índices pluviais está mais específica vista as irregularidades registradas, excetos os anos de 2011 e 2014. A curva de tendência polinomial apresenta-se com um coeficiente angular negativo e $\mathrm{R}^{2}$ de baixa significância onde não se pode afirmar que está ocorrendo aumento ou redução nos índices pluviais.

Figura 3 - Precipitação anual (mm) e sua reta de tendência polinomial, para o período de 1981-2019 no município de Lagoa Seca - PB.

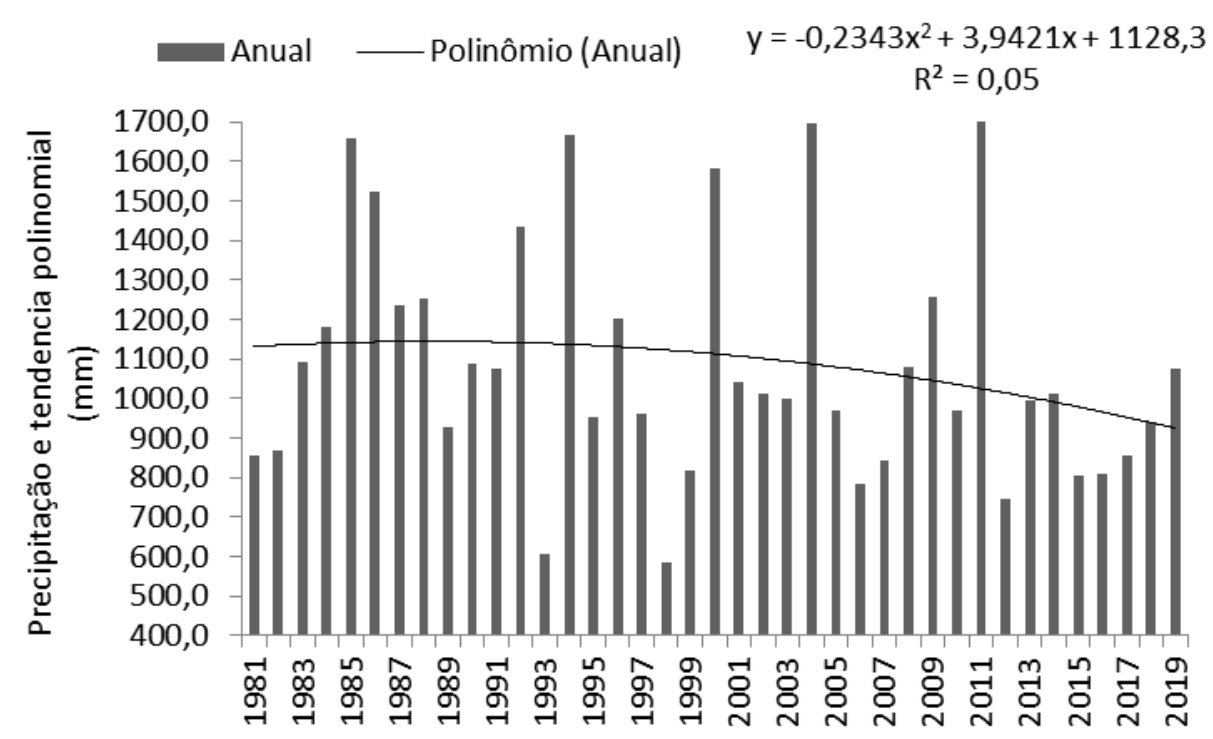

Fonte: Medeiros (2021).

Marengo et al. (2011) afirmaram que a zona de convergência intertropical (ZCIT) é o importante sistema atmosférico causador de chuvas na região nordeste do Brasil, que é representado pelo eixo do cavado equatorial e suas diferentes variações em posição e intensidade que estão correlacionadas às altas subtropicais do Atlântico Norte e Sul.

Na Figura 4 têm-se as distribuições da precipitação mensal (mm) e seus valores percentuais no período de 1981-2019 no município de Lagoa Seca - PB. Entre os meses de março a agosto os índices pluviais representam $77 \%$ do valor anual e entre os meses de setembro a fevereiro 23\%. Para Lacerda et al. (2015) cerca de 90\% dos índices pluviais da região acontecem entre os meses de janeiro a maio e no decorrer dos anos essas precipitações vêm reduzindo devido aos efeitos climáticos que ocorreram e ocorrem na escala regional, sendo o contrário para o estudo de Lacerda. 
Figura 4 - Distribuição da precipitação mensal (mm) e seus valores percentuais no período de 1981-2019 no município de Lagoa Seca - PB.

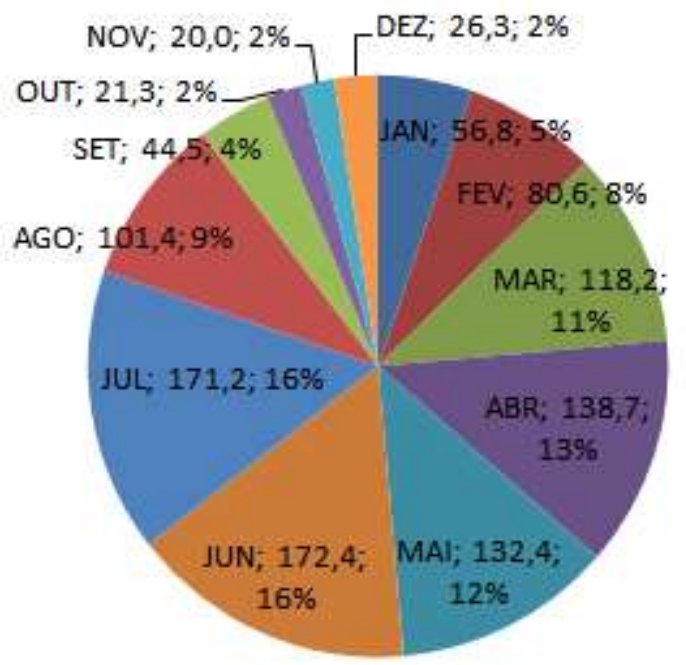

Fonte: Medeiros (2021).

Nóbrega et al. (2015), analisaram a flutuação e tendências de índices de extremos de chuva em Pernambuco por meio de dados relativos ao período de 1978 a 2010. Dantas et al. (2015) observaram tendências sazonais e anuais de índices de extremos pluviais para a Cidade de Campina Grande, no período 1975 a 2011. Esta afirmação corrobora com os resultados deste artigo.

A Figura 5 representa a precipitação observada e suas tendências de precipitações estimadas pelas médias móveis para 5 e 7 anos para a área em estudo. As estimativas das medias móveis de 7 anos apresentam valores de maiores significâncias do que para os 5 anos. Estudos semelhantes foram realizados por Galvíncio (2000) na precipitação do Rio São Francisco para (algum setor alto e médio do curso do rio).

Figura 5 - Precipitação anual e suas retas de tendência média móvel para 5 e 7 anos no período de 1981-2019 em Lagoa Seca PB.

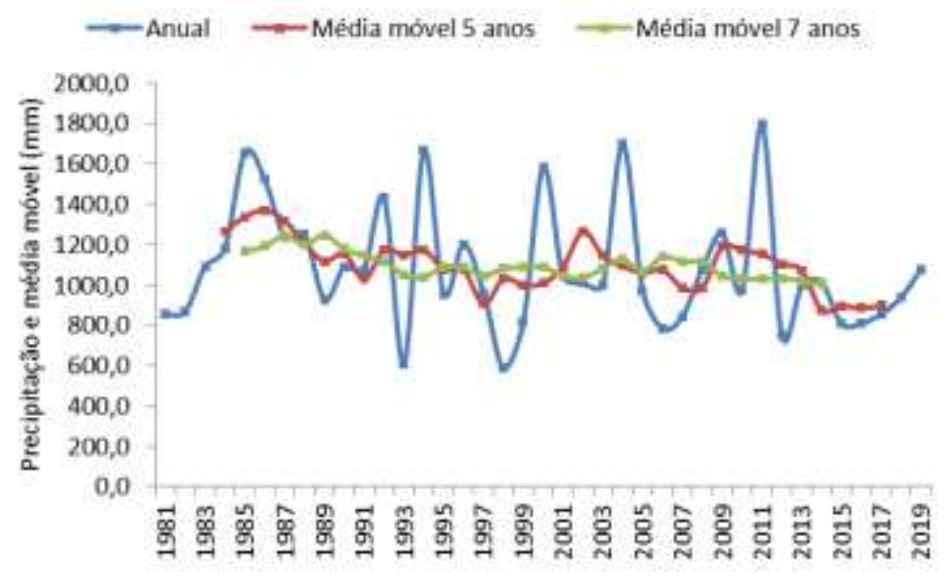

Fonte: Medeiros (2021).

Medeiros et al. (2018) mostraram que no computo das médias móveis para São Bento do Uno (PE) as estimativas das medias-móveis de 10 anos apresentam valores de maiores significâncias. Eventos extremos ocorreram em muitos casos devido 
à ocorrência simultânea de várias escalas temporais que são responsáveis por anomalias pluviométricas, corroborando com os estudos de: (Andreoli et al. 2004; Gershunov et al. 1998; Da Silva (2017).

\section{Conclusões}

Verificou-se que nas avaliações das tendências climáticas os sistemas meteorológicos que ocasionam chuvas de altas intensidades e em curtos intervalos de tempo são as formações das linhas de instabilidades, pulso isolado da ZCIT e a contribuição dos efeitos locais que colaboram para as irregularidades pluviais.

As irregularidades pluviais entre 2000-2019 registraram dez anos seco; seis anos muito seco; três anos muito chuvoso e um ano chuvoso, estas irregularidades pluviais nos leva a concluir que eventos climáticos e as ações antrópicas podem estar contribuindo para os registros destes cenários em escala local, a predominância de anos seco e muito seco vem ocasionando impactos aos produtores do hortifrutigranjeiro que vem utilizando-se de maiores tempos em irrigações.

Estudos com tendências climáticas tornam-se mais compreensível quando se analisar séries temporais longas as quais possam ser divididas em subperíodos de 30 anos ao menos.

A irregularidade no quantitativo e distribuição temporal da chuva é fatores limitantes para o desenvolvimento da produção agropecuária e hortifruticultor na área de estudo.

A série temporal constituída dos totais pluviais mensais registrados a cada ano entre 1981-2019, não representa componentes significativas de correlação serial, tendências temporais ou periodicidades.

\section{Referências}

AESA. Agencia executiva de água e clima do Estado da Paraíba. 2020.

Alvares, C. A., StaPE, J. L., Sentelhas, P. C., Gonçalves, J. L. M. \& Sparovek, G. (2014). Köppen's climate classification map for Brazil. Meteorologische Zeitschrift. 22, 711-728.

Andrade, A. J. P., Silva, N. M. \& Souza, C. R. (2014). As percepções sobre as variações e mudanças climáticas e as estratégias de adaptação dos agricultores familiares do Seridó potiguar. Desenvolvimento e Meio Ambiente 31, 77-96.

Andreoli, R. V., Kayano, M. T., Guedes, R. L., Oyama, M. D. \& Alveso, M. A. S. (2004). A influência da temperatura da superfície do mar dos Oceanos Pacífico e Atlântico na variabilidade de precipitação em Fortaleza, Revista Brasileira de Meteorologia, 19, $337-344$.

Cavalcanti, I. F. A., Nunes, L. H., Marengo, J. A., Gomes, J. L., Silveira, V. P. \& Castellano, M. S. (2017). Projections of Precipitation Changes in Two Vulnerable Regions of São Paulo State, Brazil. American Journal of Climate Change, 06, 268-293.

Cavalcanti, E. P., \& Silva, E. D. V. (1994). Estimativa da temperatura do ar em função das coordenadas locais. IN: Congresso Brasileiro de Meteorologia. 8. 1994. Belo Horizonte, Anais... SBMET, 1, 154-157.

Cavalcanti, E. P., Silva, V. P. R., \& Sousa, F. A. S. (2016). Programa computacional para estimativa da temperatura do ar. Revista Brasileira de Engenharia Agrícola e Ambiental, Brasil, 10(1), 140-147. 2006. Editora@ufcg.edu.br. p.150.

Chierice, R. A. F., \& Landim, P. M. B. (2014). Variabilidade Espaço-Temporal de Precipitação Pluviométrica na Bacia Hidrográfica do Rio Mogi Guaçu. Revista de Geociências, 33(1), 157-171.

Da Silva, D. F. (2017). Aplicação de Análises de Ondaletas para Detecção de Ciclos e Extremos Pluviométricos no Leste do Nordeste do Brasil. Revista Brasileira de Meteorologia, 32, 187-198.

Dantas, L. G., Santos, C. A. C., \& Olinda, R. A. (2015) Tendências anuais e sazonais nos extremos de temperatura do ar e precipitação em Campina Grande PB. Revista Brasileira. 20. 15-37.

Duarte, C. C., Nóbrega, R. S., \& Coutinho, R. Q. (2015). Análise climatológica e dos eventos extremos de chuva no município de Ipojuca, Pernambuco. Revista de Geografia (UFPE) 32, 158-176.

Farias, R. F. L., Alves, K. M. A. S. \& nóbrega, R. S. (2012). Climatologia da ocorrência de eventos extremos de precipitação na mesorregião do Sertão Pernambucano. Revista Geonordeste 1, 930-941.

Galvíncio, J. D. (2000). Impactos dos eventos El Niño na precipitação da Bacia do rio São Francisco. Dissertação de Mestrado em Meteorologia, Campina Grande. Universidade Federal da Paraíba (PB), p.117.

Gershunov A., \& Barnett, T. P. (1998). Interdecadal modulation of ENSO teleconnections. Bulletin of the American Meteorological Society, 79: $2715-2725$. 
Ghini, R., Hamada, E. \& Bettiol, W. (2008). Climate change and plant diseases. Scientia Agricola 65, 98-107.

Groppo, J. D., Moraes, J. M., Beduschi, C. E., Genovez, A. M. \& Martinelli, L.A. (2008). Trend analysis of water quality in some rivers with different degrees of development within the São Paulo State, Brazil. Rivers Research and Applications 1, 1-2.

IPCC. Intergovernmental Panel on Climate Change, Climate Change 2014: Synthesis Report. Contribution of Working Groups I, II and III to the Fifth Assessment Report of the Intergovernmental Panel on Climate Change. Pachauri, R.K., Meyer, L.A. (eds.). IPCC, Switzerland. 2014.

Ishihara Júnior, , Fernandes, , Duarte, A. A., AlmeidA, M., Duarte, A. R. C. 1. M., Ponte, M, X. L. \& Glauber E. Quantitative and Spatial Assessment of Precipitation in the Brazilian Amazon (Legal Amazon) - (1978 to 2007). Revista Brasileira de Recursos Hídricos, $19(1), 29$ - 39.

Karmeshu, N. (2012). Trend Detection in Annual Temperature \& Precipitation using the Mann Kendall Test - A Case Study to Assess Climate Change on Select States in the northeastern United States. Master's thesis. University of Pennslvania. Filadélfia, Penn.

Köppen, W., \& Geiger, R. (1928). Klimate der Erde. Gotha: Verlag Justus Perthes,

Köppen, W. (1931). Grundriss der Klimakunde: Outline of climate science. Berlin: Walter de Gruyter, 388p.

Lacerda, F. F., Nobre, P., Sobral, M. C. \& Lopes, G. M. B. (2015). Alterações climáticas globais: uma realidade em Pernambuco, in: Academia Pernambucana de Ciência Agronômica, Recife. 2015

Lai, C., Chen, X., Wang, Z., Wu, X., Zhao, S., Wu, X., \& Bai, W. (2016). Spatio-temporal variation in rainfall erosivity during 1960-2012 in the Pearl River Basin, China. Catena, 137, 382-391.

Marengo, J. A., Alves, L. M., Beserra, E. A., \& Lacerda, F. F. (2011). Variabilidade e mudanças climáticas no semiárido brasileiro. Recursos hídricos em regiões áridas e semiáridas. $303-422$

Marengo, J. A., \& Camargo, C. C. (2008). Surface air temperature trends in Southern Brazil for 1960 - 2002. International Journal of Climatology. 28, 893904.

Medeiros, R. M., Holanda, R. M. \& Vilar, H. N. (2018). Climate Change in Tacaimbó-PE, Brazil. Journal of Hyperspectral Remote Sensing 8, 60-66.

Medeiros, R. M., Holanda, R. M. \& Silva, V. P. (2018). Tendências pluviais e análise da média móvel para São Bento do Una - PE, Brasil. Revista de Geografia (Recife). 35(5)

Medeiros, M. C., Luz, E. L. P., Medeiros, R. M. \& Kozmhinsky, M. Variabilidade espacial e temporal da precipitação no município de Serra Talhada - PE, Brasil. 2017. I Congresso Internacional da diversidade no semiárido. Diversidade aprendendo o semiárido no semiárido e com o semiárido. Campina Grande PB. www.conidis.com.br. 2016.

Medeiros, R. M., Silva, V. M. A., Melo, V. S. \& Menezes, H. E. A. (2016). Diagnosis and trend rainfall in Bom Jesus - Piauí, Brazil. Revista verde 11, 115121.

Medeiros. R. M., Borges, C. K., Vieira, L. J. S. (2012). Precipitation climatological analysis in the municipality of Bananeiras - PB, in the period 1930-2011 as contribution to Agroindustry. Green Paper on Agroecology and Sustainable Development 2, 10.

Moura, D. M. S. (2016). Panorama geral sobre a expansão urbana e a influência nas mudanças climáticas: do âmbito mundial ao nordeste brasileiro, in: Araújo A.R., Belchior G.P.N., Viegas, T.E.S. Os impactos das mudanças climáticas no Nordeste brasileiro. Fundação Sintaf, Fortaleza, Instituto o Direito por um Planeta Verde, São Paulo, pp. 35-56.

Nobrega, R. S., Farias, R. F. L. \& Santos, C. A. C. (2015). Variabilidade Temporal e Espacial da Precipitação Pluviométrica em Pernambuco através de Indices de Extremos Climáticos. Revista Brasileira de Meteorologia, 30(2), 171 - 180.

Nóbrega, J. N., Santos, C. A. C., Gomes, O. M., Bezerra, B. G. \& Brito, J. I. B. (2014). Eventos Extremos de Precipitação nas Mesorregiões da Paraíba e suas Relações com a TSM dos Oceanos Tropicais. Revista Brasileira de Meteorologia 29, 197 - 208.

Nunes, C. R. P. (2016). As mudanças climáticas a partir da implantação de empresas de capital estrangeiro no Nordeste: Estado regulador? in: Araújo, A.R., Belchior, G.P.N., Viegas, T.E.S. (orgs). Os impactos das mudanças climáticas no Nordeste brasileiro. Fundação Sintaf, Fortaleza, Instituto O Direito por um Planeta Verde, São Paulo.

OPAS. (2014). Organização Pan-Americana da Saúde, Ministério da Saúde. Desastres Naturais e Saúde no Brasil. OPAS, Ministério da Saúde, Brasília, DF. (Série Desenvolvimento Sustentável e Saúde, 2). 2014

PBMC. (2014). Primeiro Relatório de Avaliação Nacional do Painel Brasileiro de Mudanças Climáticas, 2014. Sumário Executivo do GT2. PBMC, Rio de Janeiro, Brasil.

Penereiro, J. C., \& Orlando, D. V. (2013). Análise de tendências em séries temporais anuais de dados climáticos e hidrológicos na bacia do rio Parnaíba entre os Estados do Maranhão e Piauí/Brasil. Revista Geográfica Acadêmica 7, 5-21.

Pinkayan, S. (1966). Conditional Probabilities of Occurrence of Wet and Dry Years ver a Large Continental Area. Hidrology Papers, n. 12, Colorado State University, Boulder-Co.

Priori, J. R. L. (2014). Mudanças Climáticas e possíveis impactos nas cidades costeiras do Nordeste brasileiro. Disponível: http://research.fit.edu/sealevelriselibrary/documents/ brasileiro\%20\%28portug\%29.pdf. doc_mgr/479/Priori\%20Jr.\%202013.\%20Possiveis\%20impactos\%20no\%20nordeste\%20 
Santos, C. A. C. (2006). Estimativa e tendências de índices de detecção de mudanças climáticas com base na precipitação diária no Rio Grande do Norte e na Paraíba. Dissertação (Mestrado). Campina Grande, UFCG.

Santos Neto, L. A. (2014). Variabilidade da precipitação horária em Porto Velho - RO e suas tendências anuais e sazonais. Dissertação (Mestrado) - Programa de Pós-graduação em Desenvolvimento Regional e Meio Ambiente, UNIR, Porto Velho, Rondônia. $71 \mathrm{f} .2014$.

Thornthwaite, C. W. (1948). An Approach Toward a Rational Classification of Climate. Geogr. Ver. 38, 55-94.

Thornthwaite, C. W., \& Mather, J. R. (1955). The Water Balance. New Jersey: Drexel Institute Of Technology. Publications In Climatology, 104p.

Yang, T., Shao, Q., Hao, Z. C., Chen, X., Zhang, Z., Xu, C. Y. \& Sun, L. (2010). Regional frequency analysis and spatio-temporal pattern characterization of rainfall extremes in the Pearl River Basin, China. Journal of Hydrology, 380, 386-405, 2010.

Zhijia, G., Xingwu, D., Bing, L., Jinming, H. \& Jaonan, H. (2016). The spatial distribution and temporal variation of rainfall erosivity in the Yunnan Plateau, Southwest China: 1960-2012. 145, 291-300.

Wanderley, L. S. A., Nóbrega, R. S., Moreira, A. B., Anjos, R. S. \& Almeida, C. A. P. (2018). As chuvas na cidade de Recife: uma climatologia de extremos. Revista Brasileira de Climatologia 14, 149-164.

WMO. World Metereological Organization. Disponível: https://www.wmo.int/pages/index_en.html. 2019.

Xavier, T. M. B. S., \& Xavier, A. F. S. (1987). Classificação e Monitoração de Períodos Secos e Chuvosos e Cálculo de Índices Pluviométricos para a Região Nordeste do Brasil, Revista Brasileira de Engenharia. Cadernos de Recursos Hídricos, 5(2), 7-31.

Xavier, T. M. B. S., Silva, J. F. \& Rebello, E. R. G. A. (2002). Técnica dos quantis e suas aplicações em Meteorologia, Climatologia e Hidrologia, com ênfase para as regiões brasileiras. Thesaurus Editora de Brasília Ltda. Brasília, $141 \mathrm{p}$ 